\title{
Tenets Towards Smartphone-based Medical Tricorders
}

\author{
Colin Barry \\ c1barry@ucsd.edu \\ University of California, San Diego \\ La Jolla, CA, USA
}

\author{
Tauhidur Rahman \\ trahman@cs.umass.edu \\ University of Massachusetts \\ Amherst \\ Amherst, Massachusetts, USA
}

\author{
Edward J. Wang \\ ejaywang@eng.ucsd.edu \\ University of California, San Diego \\ La Jolla, CA, USA
}

\begin{abstract}
There is a vision for smartphone-based medical tricorders, in which a software application can be downloaded to smartphones around the world in people's pockets to use for medical sensing at the point of care. The power of this vision lies in quick software updates that could allow any phone to count coughs, track anemia, detect sleep apnea, and much more. It has the potential to enable remote healthcare effectively and equalize the reach of care. However, there is a need for further discussion around the implicit difficulties to developing and deploying medical sensing applications. The reality is that every smartphone is different, and that an application developed for one phone has no guarantee of working for the next phone or even the next OS update. In this paper, our goal is to draw out motifs around the challenges that are inherent to realizing this vision of smartphone-based medical sensing solutions. We further expound on this by juxtaposing the challenges with potential futures that we organize as tenets for how we could achieve more sustainable and universal development towards smartphone-based medical tricorders.
\end{abstract}

\section{CCS CONCEPTS}

- Human-centered computing $\rightarrow$ Smartphones; Ubiquitous and mobile computing; Ubiquitous and mobile devices; Mobile phones; Mobile devices.

\section{KEYWORDS}

Mobile Health, Smartphones, Medical Sensing, Mobile Phone, Software as a Medical Device

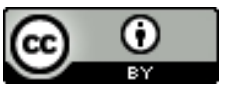

This work is licensed under a Creative Commons Attribution International 4.0 License.

DigiBiom '21, June 25, 2021, Virtual, WI, USA

(C) 2021 Copyright held by the owner/author(s).

ACM ISBN 978-1-4503-8606-7/21/06.

https://doi.org/10.1145/3469266.3469880
ACM Reference Format:

Colin Barry, Tauhidur Rahman, and Edward J. Wang. 2021. Tenets Towards Smartphone-based Medical Tricorders. In Future of Digital Biomarkers (DigiBiom '21), June 25, 2021, Virtual, WI, USA. ACM, New York, NY, USA, 7 pages. https://doi.org/10.1145/3469266. 3469880

\section{AN APPSTORE OF MEDICAL SENSORS}

The mobile health community has a vision to bring to life the science fiction of the medical tricorder, a single device that can perform a whole gamut of medical measurements. Not only that, but every person in the world could own one. The vision is grounded on the concept of leveraging sensors people already have, those built into people's personal smartphones, as the vehicle to get the sensor measurements needed for physiological calculations. Just like how an app developer could make a game and bring it to billions of people through the app store, a developer could create a software-enabled medical sensor and transform phones around the world to perform jaundice screening, anemia tracking, spirometry, sleep apnea monitoring, urine analysis, and much more.

The diversity of measures that the world needs makes the software-enabled medical sensing applications built for smartphones such an impactful concept. One of the biggest transformations in the move from feature phones to the modern smartphone is not just the touch screen, but independent app developers creating new apps for these platforms. No longer are the apps on phones restricted to what the phone manufacturers delivered with them, but new apps can be created by other app developers. This ushered in diverse user experiences driven by the collective intelligence and creativity of developers around the world. Just as we've seen in every domain of applications, from payment apps, ride hailing services, food delivery, study aids, all the way to religious worship apps, successful developers uncover needs of end users and come up with suitable solutions for the specific user group and needs. On the surface medical sensing apps may seem universal, and this may be true for a base set of metrics like blood pressure. But in reality, medical needs are very specific to different groups of people. For this reason, a pre-developed $\mathrm{N}$ number of metrics to a tricorder, like the one envisioned for the Qualcomm X-Prize in 2012 [1] may 


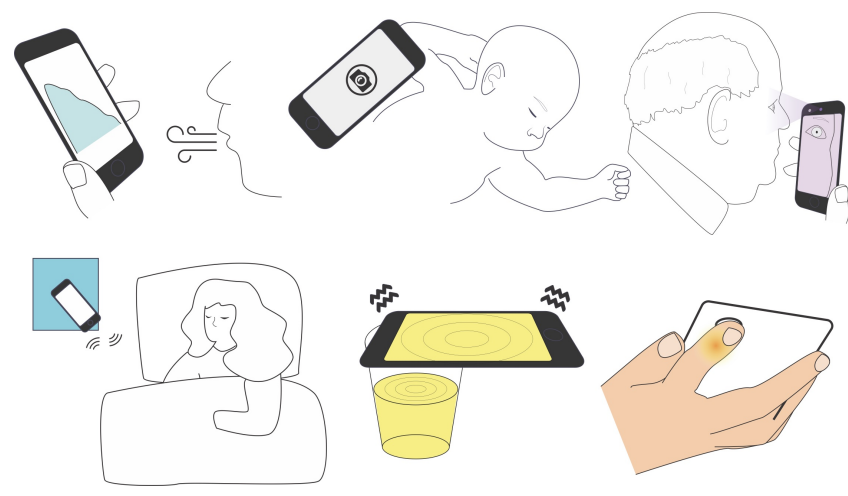

Figure 1: Smartphones have been envisioned as a readily available, ubiquitous sensor endpoint to deploy a wide range of software-enabled medical sensing for pulmonology, pediatrics, cognition, sleep, biosamples, cardiology, and much more.

not be the best solution. We instead imagine a solution that supports a diverse and inclusive body of programmers. The power of leveraging collective intelligence for developing medical sensing smartphone apps means that even those who are marginalized and not represented in the original development of the hardware platform could potentially have tailored solutions developed for their needs.

Currently, these types of software-enabled medical sensing solutions feasible for development, but not for widespread adoption. For example, any app performing measurements of pulse oxygen were removed from the app store at the height of the pandemic. These measurement apps were unregulated and billed as "for entertainment purposes only." As such, they were taken off the market to avoid misuse. In over a year of the pandemic, the closest we have is software built in-house by Google, demonstrating the simplest application of heart rate monitoring, but not $\mathrm{SpO} 2$ monitoring, blood pressure estimation, or spirometry. Thus, we need to enable medical measurements on smartphones in way that adheres with the regulatory standards of accuracy and consistency for medical devices.

\section{CHALLENGES FOR SOFTWARE-ENABLED MEDICAL SENSING ON SMARTPHONES}

Developing these software-enabled medical measurements is far from straight forward, and the physical underpinning of sensor measurements for physiologic calculations is often very device specific. Every smartphone is different, from the color of the phone and where the LED is positioned to the microphone spectral dynamics or even the set of available sensors. The reality is that each medical sensing application is really developed for a specific phone model rather than all smartphones. Not only that, smartphones, which were never developed for physiological measurements and have yet to incorporate medical sensing as a central use case, do not have the software pipeline nor the physical ergonomics to properly enable medical measurements.

\subsection{The Ever Changing Sensor Package}

Since the first iPhone introduced in 2007, most phones have had a rear camera, accelerometer, proximity sensor, vibration motor, speaker, microphone, and a screen all in about a handheld size. It of course comes as no surprise that as technology improves and demands rise, companies work to improve their product and differentiate from competitors. As such, new sensors are added year after year: GPS, front-facing camera (iPhone4/Nexus3/GalaxyS), Flash LED (iPhone3), IR blaster (GalaxyS4), 9-dof IMU, finger print readers, facial recognition IR camera (iPhoneX/Pixel4/Galaxy S9), in-display finger print reader (GalaxyS21), LIDAR (iPhone 12 Pro Max), millimeter wave gesture sensors (Pixel 4), pressure sensitive screen (Pixel 5), and more. With these new sensors come new opportunities for health sensing.

However, not all sensors introduced stay. With the introduction of facial recognition IR cameras, the need for finger print sensors was obviated (Pixel 4/iPhone 8). With new advancements in in-display fingerprint sensors, some phones are now replacing facial recognition cameras with fingerprint sensors again. Pressure sensitive screens, introduced in iPhone7, were subsequently removed in iPhone11. IR blasters caught on for a few generations in Galaxy S Series (S4-S6), but are not included in the more recent models. We may think that a sensor is now "mainstream," but companies surprise and delight users year after year with a new invention that moves the dial forward on what is now possible on phones. In a way, for regular consumers, the bigger deviation from the prior model, the more attractive and exciting the new release.

This ever evolving landscape is the economic reality of product design for consumers. But what does this mean for software-enabled medical sensing on smartphones? Moving the speaker under the display affects acoustic doppler solutions that rely on microphone and speaker alignment in-plane. This change inhibits health sensing to track chest motions for respiration and detect middle ear fluid. Removing the IR facial recognition camera eliminates the ability to conduct pupillary assessments that perform equally across lighter and darker eye colors. Improvements in low light photography causes companies to remove multiple LED lights, which destroys the possibility of using LEDs to analyze light reflection for measurements like hemoglobin concentration. 


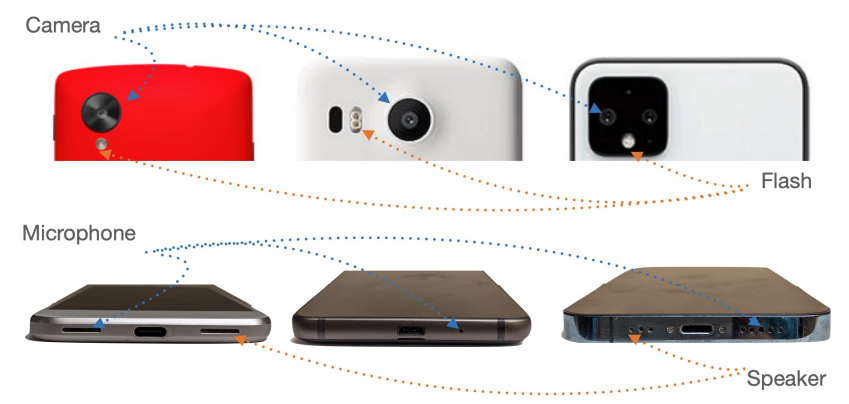

Figure 2: Hardware variations for the "same" type of sensors

\subsection{Heterogeneity of the "Same" Sensors}

Within sensors that are "mainstream," such as the camera (front and rear), microphone, speaker, and IMU, there remains heterogeneity between makes/models each year. Although many smartphones have an LED light, the type, location, and number of LED lights commonly changes. Camera lenses are different widths and distances from the LED. The camera may be positioned on the left corner of the phone or in the center. The front facing camera may be embedded in a bezel or a pinhole in the bezel-less display. Microphone openings may be slotted, pinhole, or perforated. Speakers may be positioned in the front or the bottom edge. Haptic vibrations can be made from low-cost eccentric mass motors or higher-cost linear actuator motors. Even for phones of the same model, there will be protective cases with a variety of color and structure.

These differences are designed with an explicit care to enhance the user experience, which largely relies on human perception. However, medical sensing relies heavily on the underlying physics of a measurement. If the color of the phone is different, the reflection from photoplethysmography at the fingertip results in different spectral distributions as the color of the phone is also reflected. Differences in the microphone and speaker opening will affect how the same sound may be transduced. Expiratory maneuvers such as Spirosmart [6] would be affected by changes in turbulent air flow of more perforated casings.

\subsection{APIs are Not Designed for Health Sensing}

To develop medical sensing apps, developers currently access the sensors on phones using standard APIs for making camera apps, voice recorders, game haptics, etc. Although sensor data is captured, the APIs do not allow developers to capture signals by interfacing with an imaging sensor, sound transducer, microphone, vibration motor, IMUs, etc as if they were connected to a microcontroller. Camera recordings have auto-adjust for focus, whitebalance, and exposure.
Similarly, proximity sensors only provide binary values of presence instead of sensor readings.

The data acquisition pipeline is typically asynchronous. The APIs take a command, like "record video", and set up a routine so that the frames of the video come back based on the parameters submitted. However, the timing of when the routine actually starts is not guaranteed. Also, sampling rate may not always be consistent and can be throttled based on processor load balancing or temperature management.

This lack of control is particularly aggravated when utilizing multiple sensor subsystems. For example, recording to pulse transit time for blood pressure estimation relies on capturing the timing difference between the heart beat and the arrival of the pulse at the finger. To measure this, solutions use the microphone or accelerometer to capture the beat of the heart and the camera to capture the pulse at the finger. Experiments done in [4] show a two frame delay between microphone and camera systems. In the author's own experience, the delay is inconsistent between phone models and even recordings. The inconsistent time delay between the IMU system and the camera system is even less controlled [11].

\subsection{Physical "Impedance" Mismatch}

How a medical device will interface with the human body is typically one of the first design considerations in the development process. In the same way, the shape of the phone is also designed for the way it will interface with the human body based on typical use cases. The phone is easy to carry and effectively designed for phone calls with the speaker held up to the ear and microphone at the mouth (Fig 3 a). The screen and speakers are suitable for media viewing away from the user (Fig $3 \mathrm{~b}$ ). The phone, however, is not designed to be pressed on the chest, have the finger covering the camera and LED, record sound from body cavities, and block ambient light out when taking pictures of the skin and eyes.

In comparison, medical devices are designed with physical interfaces that match the specific sensing needs: (Fig $3 \mathrm{c}$ ) pulse oximeter with finger clip, (Fig $3 \mathrm{~d}$ ) spirometer with nose clip and mouth funnel, (Fig 3 e) otoscope with light and ear piece, (Fig $3 \mathrm{f}$ ) opthalmoscope with eye cup, (Fig 3 g) stethoscope with diaphram, and (Fig $3 \mathrm{~h}$ ) bilirubinometer with small point for skin contact

To address these issues, passive attachments have often been pointed to as solutions to transduce and augment the sensors of smartphones to better interface with the human body. Examples include a funnel for sound channeling [2], vortex whistle to create pitch for wind velocity [5], finger holder to provide mechanical support [13], eye piece to block external light [9], and a color reference card for lighting correction $[3,7,8,10]$. However, these attachments can hinder 

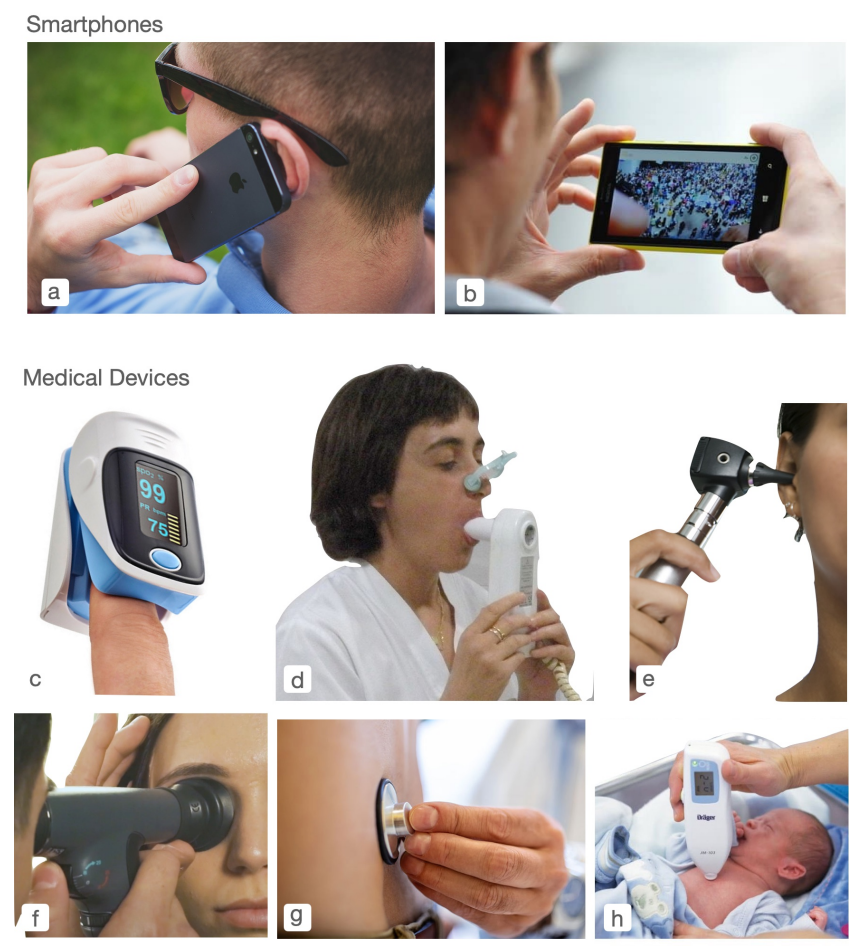

Figure 3: (Top) Smartphone are designed to accommodate voice calls and media consumption, whereas (Bottom) medical device have physical ergonomics that conform to the body part to be measured and support signal transduction.

the ubiquity of the measurement because they are often not widespread nor universal across phone models.

\section{TENETS TOWARDS MAKING THE SMARTPHONE TRICORDER A REALISTIC FUTURE}

The onus of making software-enabled medical sensing solutions functional have largely been placed on the developers. But as we point out, the various challenges developers face with smartphone development render this extremely challenging within the economic realities of the smartphone ecosystem. This kind of flux may be manageable for entertainment, productivity, and creativity apps, but the potential that an OS update, another background app running, or a different camera position could result in an inaccurate or obsolete medical measurement, is a non-starter. Beyond surfacing the list of challenges, the central goal of this paper is to point out a set of basic tenets for pushing the vision of smartphone-based medical tricorders towards a usable reality.

In drafting these tenets, we accept the fact that it is not a solution to simply suggest that smartphones should all be the same. We do need to live within the realities that smartphones are different, will change, and won't be shaped like a medical device. However, that does not mean it is infeasible to create more suitable APIs, development pipelines, and ultra-low cost, widespread attachments.

\subsection{Algorithms Resilient to Slight Hardware Variations}

One of the realities of developing on smartphone devices is that there will inevitably be some level of hardware variations across models. This is particularly important with the use of machine learning backed algorithms. One of the advantages of using machine learning to perform the physiological measures is that small variations could be introduced in the training data. This may come in two forms. The first is capturing fundamental phenomenon that are largely hardware independent. The second is relying on computational model architectures that explicitly aim to learn the underlying representation for the measurement separately from the hardware.

For optical measures on cameras, the recording of shapes or changes in the absorption across time in the same color channel is often a robust measurement. In comparison, comparing color measurements across color channels is difficult due to whitebalance/illumination conditions. For acoustic measures on microphones, the presence of a frequency band is particularly well preserved across hardware. This also extends to capturing shifts in sound frequencies. On the other hand, the frequency response of microphones is not flat across a frequency band; it depends on the microphone model and enclosure. Thus, exact amplitude comparisons across frequency bands may not be reliable across different devices. In addition, acoustics closer to the device are influenced more by the exact position of the microphone. For instance, turbulent air at the microphone opening can create broadband noise. External vibration and motion measurements are reliable with IMUs (e.g. limb movements). However, measures that rely on capturing the the device's vibration, like Vibroscale liquid measurements [14], are much more device dependent based on the type of motor creating the vibration (eccentric vs linear actuator motor) and the resonance of the device, which depends on the size and material of the phone construction.

We point out measures that are less resilient to hardware variations, not as examples of physical phenomenon to avoid when developing smartphone-based medical sensing applications, but rather as measures that require extra care when developing. For example, to leverage ratiometric measurements in optical systems, the whitebalance and illumination cannot be ignored and could be supported using a color reference. Recent developments in the use of metalearning architecture 


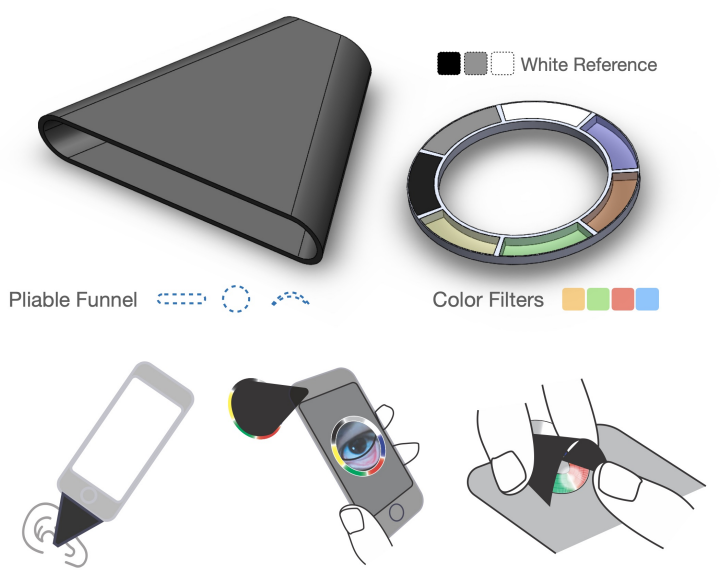

Mixable \& Conformable for the Application

Figure 4: Conceptual multipurpose smartphone attachment

for model training may also provide another tool for creating more resilient solutions. A potential mechanism could be to capture the response of the same specimen with different phone setups to train multiple parallel models and utilize metalearning to distill a higher level model that potentially nullifies the effect of specific hardware configurations.

\subsection{Ultra-low Cost, Repurposable Accessories}

Passive attachments are a common approach to adjust the phone's sensors to better interface the phone to the body or to transduce the physical phenomenon to one that is measurable by standard phone sensors. Although it is not very viable to dictate where the sensors should be positioned on the phone and how the physical enclosure of the phone will be designed, an avenue that can be designed are accessories for the phone. The key here is to create a small set of really cheap accessories that could potentially be massively distributed and are not for a single purpose. Figure 4 exemplifies a conceptual design of one such multi-purpose accessory that can mix-and-match to accommodate a wide variety of usecases. The conceptual accessory consists of (Top Left) a semi-rigid pliable funnel and (Top Right) a color reference and filter ring. By shaping the funnel and mixing and matching the two components, one can imagine a variety of use cases: (Bottom Left) acoustic guide for inner ear measurement, (Bottom Center) color correction/ambient light removal for camera measurement, and (Bottom Right) LED color modification through red/green narrow band filters for finger cuff $\mathrm{SpO} 2$.

\subsection{APIs that Prioritize Sensor Data Flow Over User Interface}

An API that is more suitable for medical sensing application development should focus on providing control over sampling rate, retrieval of both minimally processed and filtered signals, modulation of actuators, and synchronization of sensor/actuator systems.

At the hardware level, all of the above mentioned is certainly possible. The challenge is around creating the interfaces at the firmware level. In [12], Wang et al. show that the proximity sensor on the phone can operate as an IR sensor when modifications are made to the firmware to readout the raw sensor readings through the interface debug mode. There have been APIs created for better interfacing with sensor systems to allow degrees of control more suitable for the application. The Android Camera2 API, for example, was designed specifically to give developers the ability to make applications that can control camera systems, much like a SLR camera. The API is setup for applications to set and fix focus, whitebalance, and exposure.

\subsection{Sustainable Hardware Evolution, Differentiation, and Documentation}

Another way to look at the research findings thus far is that low tolerance sensors on smartphones CAN provide the sensor measurements for a wide variety of measures. The challenge is that phones are different and thus it is difficult to make sure any of the sensing solutions can indeed work. But for the most part, low-cost phones have the sensors needed to achieve this vision.

The different tenets thus far take an assumption that phones in the future will remain diverse. However, it is possible to imagine a low-cost smartphone line standardized specifically for medical sensing applications. Such a solution may not be equivalent to the idea that "any smartphone in anyone's pocket" can transform into a medical tricorder, but could still fill a much needed healthcare need for lowresource clinics and mobile care scenarios. A pop-up or lowresource clinic, community center in a remote village/town, traveling health provider, school nurse and coaches, and any scenario where a dedicated device that costs similarly to a low-end smartphone of about $\$ 100-\$ 200$ can provide access to a broad library of monitoring.

In this tenet, our main focus is not necessarily what should be the blueprint for such a phone. A viable design could be to take any white label phone under $\$ 200$ and create a Health Sensing API and a multi-purpose attachment mentioned in previous tenets. The focus, rather, is about how such a line of smartphones should continue to improve. 
Drawing from other domains where standardization has led to proliferation like operating systems, computer architecture (i.e. RISC), and programming languages, we see that improvements continue to occur. However, changes, such as an added feature, are consciously designed so that long-term supported functionalities are not impacted. Right now, this same consideration is made in smartphone designs, not for medical sensing, but for user interaction, communication, photography, etc. For the medical smartphone line, the focus would instead turn to medical sensing as the primary use case to keep it consistent. With a dedicated design used for medical sensing, clear documentation of the kinds of sensors, their placement, and specifications should also be available to developers. Likely, a way this would occur would be a more spaced out release cycle between major releases. The key is to sustainably evolve the hardware as more advanced sensors today become cheaper in the future, without creating a wake of non-functioning applications that require companies to complete a whole new FDA process.

For example, things that could still distinguish between higher end models and lower end models could be the processing unit. This would likely not affect most of the applications in terms of the physical measurement, but it may create a better user experience through faster results. In this way, the regular releases of non-medical smartphones, a testing ground of new sensors, can be clearly thought of as a place where researchers could still experiment with new health sensing solutions. This allows for suggesting new directions and providing evidence that a new sensor or configuration could be rolled into the medical smartphone. A clear understanding can be delineated that unless an app is developed on the standard of medical smartphone hardware, it is not guaranteed to work.

\section{FUTURE STEPS TOWARDS A SMARTPHONE MEDICAL TRICORDER}

In this paper, we present challenges that currently impede the vision of the medical tricorder and propose tenants as potential futures for the community to improve proliferation of smartphone-based health sensing. In concluding, we present areas for future investigation.

\subsection{Extending the Scope of Research to Other Ubiquitous Devices}

While this paper focuses around extending the health sensing capabilities of smartphones, the growing ubiquity of other mobile devices with embedded sensors presents similar opportunities. At the moment, access to many of the raw sensor streams on wearable and smart home devices are quite limited. Even so, the challenges to smartphone-based health sensing also apply to these other types of devices.

\subsection{Extending Research Investigations Beyond One Phone}

An important question the research community faces moving forward is whether demonstrating functionality of "smartphonebased" health sensing solutions on a single phone model is sufficient. Researchers do not have the inside knowledge around product roadmaps and cannot control the diversity of products produced by every company. However, as new sensors arrive on phones, a type of research inquiry exists around whether this new sensor has health sensing potential. But as discussed, these sensors often depreciate from the lineup the next year. A reasonable criticism is then, if a sensor setup only exists on a few high end phones, then the proposed solution is no less custom as a dedicated device.

Should the research community then focus on solutions that are applicable for multiple phones? This includes working only with a small set of sensors that have been tried and true, and providing validation on a set of phones that are the most "standard." For example, a spirometry application could be validated on multiple phones with different microphone arrangements. This will put the onus on researchers to pursue applications that generalize well and may lead to more replicable research artifacts.

Alternatively, should the research community work towards creating a research platform for smartphone-based health sensing development? Much like how the USRP is the defacto for the wireless sensing community, some sort of white label phone could be the canonical phone agreed upon by researchers, industry, and regulatory bodies. This way, the canonical design would be updated transparently based on input from a panel. Also, translating a solution to a new phone would be a much more straight forward process. If a company wants to enable health sensing in a specific line-up of phones, they leverage the features of the cannonical phone design.

\subsection{Engaging Regulators and End Users}

Solving the challenges we present in this paper obligates a larger prospective than solely that of research scientists and engineers. To fully understand the problems and begin working towards effective solutions will require collaboration with domain experts outside of academia. The ideas and concerns of medical experts, FDA regulators, economists, NGOs, hospital administrators, and others should be considered to highlight the goals and potential pitfalls that may be overlooked. In the coming months, we hope to continue to improve upon our work here with a more inclusive perspective. We plan to start a multifaceted conversation on the challenges and potential solutions of achieving viable and accessible medical applications on a variety of smartphones. 


\section{REFERENCES}

[1] 2021. Tricorder X Prize. https://en.wikipedia.org/w/index.php?title= Tricorder_X_Prize\&oldid=1004443454 Page Version ID: 1004443454.

[2] Justin Chan, Sharat Raju, Rajalakshmi Nandakumar, Randall Bly, and Shyamnath Gollakota. 2019. Detecting middle ear fluid using smartphones. Science Translational Medicine 11, 492 (May 2019). https://doi.org/10.1126/scitranslmed.aav1102 Publisher: American Association for the Advancement of Science Section: Research Article.

[3] Lilian de Greef, Mayank Goel, Min Joon Seo, Eric C. Larson, James W. Stout, James A. Taylor, and Shwetak N. Patel. 2014. Bilicam: using mobile phones to monitor newborn jaundice. In Proceedings of the 2014 ACM International foint Conference on Pervasive and Ubiquitous Computing (UbiComp '14). Association for Computing Machinery, New York, NY, USA, 331-342. https://doi.org/10.1145/2632048.2632076

[4] Alair Dias Junior, Srinivasan Murali, Francisco Rincon, and David Atienza. 2015. Estimation of Blood Pressure and Pulse Transit Time Using Your Smartphone. In 2015 Euromicro Conference on Digital System Design. 173-180. https://doi.org/10.1109/DSD.2015.90

[5] Spencer Kaiser, Ashley Parks, Patrick Leopard, Charlie Albright, Jake Carlson, Mayank Goel, Damoun Nassehi, and Eric C. Larson. 2016 Design and learnability of vortex whistles for managing chronic lung function via smartphones. In Proceedings of the 2016 ACM International Joint Conference on Pervasive and Ubiquitous Computing (UbiComp '16). Association for Computing Machinery, New York, NY, USA, 569-580. https://doi.org/10.1145/2971648.2971726

[6] Eric C. Larson, Mayank Goel, Gaetano Boriello, Sonya Heltshe, Margaret Rosenfeld, and Shwetak N. Patel. 2012. SpiroSmart: using a microphone to measure lung function on a mobile phone. In Proceedings of the 2012 ACM Conference on Ubiquitous Computing (UbiComp '12). Association for Computing Machinery, New York, NY, USA, 280-289. https://doi.org/10.1145/2370216.2370261

[7] Robert G. Mannino, David R. Myers, Erika A. Tyburski, Christina Caruso, Jeanne Boudreaux, Traci Leong, G. D. Clifford, and Wilbur A. Lam. 2018. Smartphone app for non-invasive detection of anemia using only patient-sourced photos. Nature Communications 9, 1 (Dec. 2018), 4924. https://doi.org/10.1038/s41467-018-07262-2 Number: 1 Publisher: Nature Publishing Group.

[8] Alex Mariakakis, Megan A. Banks, Lauren Phillipi, Lei Yu, James Taylor, and Shwetak N. Patel. 2017. BiliScreen: Smartphone-Based Scleral Jaundice Monitoring for Liver and Pancreatic Disorders. Proceedings of the ACM on Interactive, Mobile, Wearable and Ubiquitous Technologies 1, 2 (June 2017), 20:1-20:26. https://doi.org/10.1145/3090085

[9] Alex Mariakakis, Jacob Baudin, Eric Whitmire, Vardhman Mehta, Megan A. Banks, Anthony Law, Lynn Mcgrath, and Shwetak N. Patel. 2017. PupilScreen: Using Smartphones to Assess Traumatic Brain Injury. Proceedings of the ACM on Interactive, Mobile, Wearable and Ubiquitous Technologies 1, 3 (Sept. 2017), 81:1-81:27. https: //doi.org/10.1145/3131896

[10] Sang Mok Park, Michelle A. Visbal-Onufrak, Md Munirul Haque, Martin C. Were, Martin C. Were, Martin C. Were, Violet Naanyu, Md Kamrul Hasan, Young L. Kim, Young L. Kim, Young L. Kim, and Young L. Kim. 2020. mHealth spectroscopy of blood hemoglobin with spectral super-resolution. Optica 7, 6 (June 2020), 563-573. https://doi.org/10.1364/OPTICA.390409 Publisher: Optical Society of America.

[11] Edward Jay Wang, Junyi Zhu, Mohit Jain, Tien-Jui Lee, Elliot Saba, Lama Nachman, and Shwetak N. Patel. 2018. Seismo: Blood Pressure Monitoring using Built-in Smartphone Accelerometer and Camera. In Proceedings of the 2018 CHI Conference on Human Factors in Computing Systems (CHI '18). Association for Computing Machinery, New York, NY, USA, 1-9. https://doi.org/10.1145/3173574.3173999
[12] Edward Jay Wang, Junyi Zhu, William Li, Rajneil Rana, and Shwetak Patel. 2017. HemaApp IR: noninvasive hemoglobin measurement using unmodified smartphone cameras and built-in LEDs. In Proceedings of the 2017 ACM International foint Conference on Pervasive and Ubiquitous Computing and Proceedings of the 2017 ACM International Symposium on Wearable Computers (UbiComp '17). Association for Computing Machinery, New York, NY, USA, 305-308. https://doi.org/10.1145/ 3123024.3123180

[13] Christopher M Westgard. 2017. Lessons Learned From Field Deployment of the Hemaapp: A Non-Invasive Hemoglobin Measurement tool. Biomedical fournal of Scientific \& Technical Research 1, 7 (Dec. 2017). https://doi.org/10.26717/BJSTR.2017.01.000569

[14] Shibo Zhang, Qiuyang Xu, Sougata Sen, and Nabil Alshurafa. 2020. VibroScale: turning your smartphone into a weighing scale. In $A d$ junct Proceedings of the 2020 ACM International foint Conference on Pervasive and Ubiquitous Computing and Proceedings of the 2020 ACM International Symposium on Wearable Computers (UbiComp-ISWC '20). Association for Computing Machinery, New York, NY, USA, 176-179. https://doi.org/10.1145/3410530.3414397 